\title{
Cylindrical symmetry: II. The Green's function in 3+ 1 dimensional curved space
}

\author{
Gopinath Kamath ${ }^{1, *}$ \\ ${ }^{1}$ Department of Mathematics, Indian Institute of Technology Tirupati, \\ Renigunta Road, Tirupati 517 506, India
}

\begin{abstract}
An exact solution to the heat equation in curved space is a much sought after; this report presents a derivation wherein the cylindrical symmetry of the metric $g_{\mu v}$ in $3+1$ dimensional curved space has a pivotal role. To elaborate, the spherically symmetric Schwarzschild solution is a staple of textbooks on general relativity; not so perhaps, the static but cylindrically symmetric ones, though they were obtained almost contemporaneously by H. Weyl, Ann. Phys. Lpz. 54, 117 (1917) and T. Levi-Civita, Atti Acc. Lincei Rend. 28, 101 (1919). A renewed interest in this subject in C.S. Trendafilova and S.A. Fulling, Eur.J.Phys. 32, 1663(2011) - to which the reader is referred to for more references motivates this work, the first part of which (cf.Kamath, PoS (ICHEP2016) 791) reworked the Antonsen-Bormann idea - arXiv:hep-th/9608141v1 that was originally intended to compute theheat kernel in curved space to determine - following D.McKeon and T.Sherry, Phys. Rev. D 35, 3584 (1987) - the zeta-function associated with the Lagrangian density for a massive real scalar field theory in $3+1$ dimensional stationary curved space to one-loop order, the metric for which is cylindrically symmetric. Using the same Lagrangian density the second part reported here essentially revisits the second paper by Bormann and Antonsen arXiv:hep 9608142v1 but relies on the formulation by the author in S. G. Kamath, AIP Conf.Proc.1246, 174 (2010) to obtain the Green's function directly by solving a sequence of first order partial differential equations that is preceded by a second order partial differential equation.
\end{abstract}

\section{Introduction}

This is the second of two papers associated with the Lagrangian density

$$
L=\frac{1}{2} g^{\mu v} \partial_{\mu} \phi \partial_{\nu} \phi-\frac{1}{2} m^{2} \phi^{2}
$$

\footnotetext{
*E-mail: gkamath01865@gmail.com
} 
for a real massive scalar field $\phi$ in $3+1$ dimensional curved space; it extends the scope of the first[1] to obtain the Green's function $G\left(x, x^{\prime} ; \sigma\right)$ as a solution to

$$
B G=-\frac{\partial G}{\partial \sigma}
$$

using a method worked out previously [2] and presented briefly further below. The operator $B$ In eq.(2) is defined from (1) as

$$
B \equiv-\partial^{\mu}\left(g_{\mu \nu} \partial^{v}\right)-m^{2}
$$

and $G$ is subject to the initial condition $G\left(x, x^{\prime} ; \sigma \rightarrow 0\right)=\delta^{(4)}\left(x-x^{\prime}\right)$.

In this effort one begins with:

a. A write of $G\left(x, x^{\prime} ; \sigma\right)$ as $G\left(x, x^{\prime} ; \sigma\right)=G_{0} e^{-T}$ where $G_{0}$ - the Green's function in flat space - is a solution to

$$
\left(-\eta^{a b} \partial_{a} \partial_{b}-m^{2}\right) G_{0}=-\frac{\partial G_{0}}{\partial \sigma} \text { subject to } G_{0}\left(x, x^{\prime} ; \sigma \rightarrow 0\right)=\delta^{(4)}\left(x-x^{\prime}\right),
$$
its

Euclidean version being

$$
G_{0}=(4 \pi \sigma)^{-2} e^{-\frac{\left(x-x^{\prime}\right)^{2}}{4 \sigma}-m^{2} \sigma}
$$

with $\eta^{a b}=\operatorname{diag}(1-1-1-1)$.

b. Now representing $T$ as $T=\frac{\tau_{-1}}{\sigma}+\sum_{k=1}^{\infty} \tau_{k} \sigma^{k}$, makes $\tau_{-1}$ a solution to

$$
\partial^{a} \partial_{a} \tau_{-1}-2 \partial^{a} \tau_{0} \partial_{a} \tau_{-1}-\left(x-x^{\prime}\right)^{a} \partial_{a} \tau_{0}=0
$$

with $\tau_{0}$ defined further below; equally, one gets:

$$
2 \partial^{a} \tau_{-1} \partial_{a} \tau_{-1}+\left(x-x^{\prime}\right)^{a} \partial_{a} \tau_{1}-f+\tau_{1}+\partial^{a} \tau_{0} \partial_{a} \tau_{0}-\partial^{a} \partial_{a} \tau_{0}=0
$$

and

$$
2 \partial^{a} \tau_{-1} \partial_{a} \tau_{2}+\left(x-x^{\prime}\right)^{a} \partial_{a} \tau_{1}+2 \tau_{2}+2 \partial^{a} \tau_{1} \partial_{a} \tau_{0}-\partial^{a} \partial_{a} \tau_{1}=0
$$

for $\tau_{1}$ and $\tau_{2}$ respectively, with similar coupled partial differential equations for $\tau_{n}$, $n \geq 3$. While eq.(5) is a second order partial differential equation for $\tau_{-1}$, eqs.(6) and (7) - as well as those defining the subsequent $\tau_{n}$ - are of the first order thus allowing for an easy solution.

\section{Calculating $\mathbf{G}$}

\section{$2.12+1$ dimensions}

As a prelude to determining $G$ it pays to start with $2+1$ dimensional curved space below , the metric for which is given by $[3,4]$ 
$g_{00}=1, g_{01}=-\frac{\lambda y}{r^{2}}, g_{02}=\frac{\lambda x}{r^{2}}, g_{11}=-1+\left(\frac{\lambda y}{r^{2}}\right)^{2}, g_{12}=-\frac{\lambda^{2} x y}{r^{2}}, g_{22}=-1+\left(\frac{\lambda x}{r^{2}}\right)^{2}$

with $2 \pi \lambda=\kappa J, \kappa=8 \pi G, G$ being the gravitational constant and $J=|\vec{J}|$ being the spin of the massless particle located at the origin[5]. Eq.(8) enables one to deduce

the vierbeins $e_{\mu}^{a}$ from $g_{\mu \nu}=\eta_{a b} e_{\mu}^{a} e_{v}^{b}$ and a convenient set is:

$e_{\mu}^{a}: e_{0}^{0}=1, e_{0}^{1}=0=e_{0}^{2} ; e_{1}^{0}=-\frac{\lambda y}{r^{2}}, e_{1}^{1}=\frac{1}{\sqrt{2}}=-e_{1}^{2} ; e_{2}^{0}=\frac{\lambda x}{r^{2}}, e_{2}^{1}=-\frac{1}{\sqrt{2}}=e_{2}^{2}$

with their inverses $e_{a}^{\mu}$ determined from $e_{\mu}^{a} e_{b}^{\mu}=\delta_{b}^{a}$ as:

$$
e_{a}^{\mu}: e_{0}^{0}=\frac{i \lambda y}{r^{2}}, e_{0}^{1}=i, e_{0}^{2}=0 ; e_{1}^{0}=i, e_{1}^{1}=0=e_{1}^{2} ; e_{2}^{0}=\frac{\lambda x}{r^{2}}, e_{2}^{1}=0, e_{2}^{2}=-1
$$

From eqs.(10) one gets with $g^{\mu v}=\eta^{a b} e_{a}^{\mu} e_{b}^{v}, \eta^{a b}=\operatorname{diag}(1-1-1-1)$ the following:

$g^{00}=1-\frac{\lambda^{2}}{r^{2}}, g^{01}=-\frac{\lambda y}{r^{2}}, g^{02}=\frac{\lambda x}{r^{2}}, g^{11}=-1, g^{12}=0, g^{22}=-1$

With the vierbeins in (9) and (10) and the definition of $\tau_{0}=-\frac{1}{2} \int e_{\alpha}^{n} \partial_{n}\left(e_{k}^{\alpha}\right) d x^{k}$ as in Ref.2 it is easy to check that $\tau_{0}=0$. Eq.(5) now becomes $\partial_{a} \partial_{a} \tau_{-1}=0$ in 3 - dimensional Euclidean space, leading to $\tau_{-1}=\frac{C}{R}$ with $R=\sqrt{\left(x_{0}^{2}+x_{1}^{2}+x_{2}^{2}\right)}$ and $C$ an integration constant. Again, with eqs.(9) and (10) and

$$
f=\frac{1}{2} \eta^{a b} \partial_{a}\left(e_{\alpha}^{m} \partial_{m}\left(e_{b}^{\alpha}\right)\right)+\frac{1}{4} \eta^{a b}\left(e_{\alpha}^{m} \partial_{m}\left(e_{a}^{\alpha}\right)\right)\left(e_{\alpha}^{m} \partial_{m}\left(e_{b}^{\alpha}\right)\right)
$$

as in Ref.2 one gets $f=0$ thereby reducing eq.(6) to

$$
2 \partial^{a} \tau_{-1} \partial_{a} \tau_{-1}+\left(x-x^{\prime}\right)^{a} \partial_{a} \tau_{1}+\tau_{1}=0
$$

whose solution in Euclidean space with $x^{\prime}=0$ is

$$
\tau_{1}=D\left(2 C-R^{3}\right)^{-1 / 3}
$$

with another integration constant $D$.It is now easy to solve eq.(7) with $\tau_{0}=0$, the answer being

$$
\tau_{2}=C_{1} \tau_{1}^{2}-\frac{24 C}{5 D^{5}} \tau_{1}^{2}\left(R \tau_{1}^{5}-\int d R \tau_{1}^{5}\right)
$$

taking $x^{\prime}=0$ in eq.(7), $C_{1}$ being an integration constant.

\section{$2.23+1$ dimensions}

It is tantalizing to assume that eqs.(5) - (7) will be just as easy to solve in $3+1$ dimensions for which the metric[1] is taken as 


$$
\begin{aligned}
& g_{00}=\left(\frac{a}{r}\right)^{4}, g_{11}=-\frac{1}{r^{2}}\left(y^{2}+\frac{a^{8} x^{2}}{r^{8}}\right), g_{22}=-\frac{1}{r^{2}}\left(x^{2}+\frac{a^{8} y^{2}}{r^{8}}\right), \\
& g_{12}=-\frac{x y}{r^{2}}\left(1-\frac{a^{8}}{r^{8}}\right), g_{33}=-\left(\frac{a}{r}\right)^{4}
\end{aligned}
$$

with $a$ a non-zero constant, the remaining $g_{i j}, i \neq j$ in (16) being zero. For eqs.(16) it is convenient to adopt for the calculation here the vierbeins given by

$$
\begin{gathered}
e_{0}^{0}=0, e_{0}^{1}=\frac{i a^{2}}{\sqrt{2} r^{2}}, e_{0}^{2}=-\frac{i a^{2}}{\sqrt{2} r^{2}}, e_{0}^{3}=0 ; e_{1}^{0}=\frac{i a^{4} x}{r^{5}}, e_{1}^{1}=0=e_{1}^{2}, e_{1}^{3}=\frac{y}{r} ; \\
e_{\mu}^{a}: \\
e_{2}^{0}=\frac{i a^{4} y}{r^{5}}, e_{1}^{1}=0=e_{1}^{2}, e_{1}^{3}=-\frac{x}{r} ; e_{3}^{0}=0, e_{3}^{1}=\frac{a^{2}}{\sqrt{2} r^{2}}, e_{3}^{2}=\frac{a^{2}}{\sqrt{2} r^{2}}, e_{3}^{3}=0
\end{gathered}
$$

$e_{a}^{\mu}:$

$$
\begin{aligned}
& e_{0}^{0}=0, e_{0}^{1}=-\frac{i x r^{3}}{a^{4}}, e_{0}^{2}=-\frac{i y r^{3}}{a^{4}}, e_{0}^{3}=0 ; e_{1}^{0}=-\frac{i r^{2}}{\sqrt{2} a^{2}}, e_{1}^{1}=0=e_{1}^{2}, e_{1}^{3}=\frac{r^{2}}{\sqrt{2} a^{2}} \\
& e_{2}^{0}=-\frac{i r^{2}}{\sqrt{2} a^{2}}, e_{2}^{1}=0=e_{2}^{2}, e_{2}^{3}=\frac{r^{2}}{\sqrt{2} a^{2}} ; e_{3}^{0}=0, e_{3}^{1}=\frac{y}{r}, e_{0}^{2}=-\frac{x}{r}, e_{0}^{3}=0
\end{aligned}
$$

Eqs.(17) and(18) yield

$$
\tau_{0}=-\log r, f=\frac{1}{r^{2}}
$$

thus making the solution of eqs.(5) - (7) less accessible relative to the $2+1$ dimensional case . Yet, it pays to rework eq.(5) to the form given in Ref.2 but in Euclidean space, viz.

$$
\partial_{a} \partial_{a} \chi_{-1}+\left(\partial_{a} \partial_{a} \tau_{0}-\partial_{a} \tau_{0} \partial_{a} \tau_{0}\right) \chi_{-1}=\left(x-x^{\prime}\right)_{a} \partial_{a} \tau_{0} e^{-\tau_{0}}
$$

using the definition $\tau_{-1}=\chi_{-1} e^{\tau_{0}}$.

With eq.(19),(20) becomes with $x^{\prime}=0$,a second order inhomogeneous partial differential equation viz.

$$
\partial_{a} \partial_{a} \chi_{-1}-\frac{1}{r^{2}} \chi_{-1}=-r
$$

for which a solution via a Green's function suggests itself. To do so one starts with

$$
\left(\partial_{0}^{2}+\partial_{z}^{2}+\frac{1}{r} \frac{\partial}{\partial r}\left(r \frac{\partial}{\partial r}\right)+\frac{1}{r^{2}} \frac{\partial^{2}}{\partial \theta^{2}}-\frac{1}{r^{2}}\right) G=-\frac{\delta\left(r-r^{\prime}\right)}{r} \delta\left(\theta-\theta^{\prime}\right) \delta\left(z-z^{\prime}\right) \delta\left(x_{0}-x_{0}^{\prime}\right)
$$

implying that

$$
\chi_{-1}=\int d x_{0}^{\prime} d z^{\prime} d \theta^{\prime} d r^{\prime} G\left(x_{0} z \theta r \mid x_{0}^{\prime} z^{\prime} \theta^{\prime} r^{\prime}\right) r^{\prime}
$$

with the Fourier transform $G=\left(\frac{1}{2 \pi}\right)^{2} \int_{-\infty}^{\infty} d s d \omega e^{i \omega x_{0}^{\prime}+i s z^{\prime}} H(r, \theta, s, \omega)$ 
Following Duffy[6], eq.(23) leads from (22) to

$$
\left(\frac{1}{r} \frac{\partial}{\partial r}\left(r \frac{\partial}{\partial r}\right)+\frac{1}{r^{2}} \frac{\partial^{2}}{\partial \theta^{2}}-\left(s^{2}+\omega^{2}+\frac{1}{r^{2}}\right)\right) H=-\frac{\delta\left(r-r^{\prime}\right)}{r} \delta\left(\theta-\theta^{\prime}\right) e^{-i s z-i \omega x_{0}}
$$

Going further[6] one gets to a Bessel - like equation for (24) viz.

$$
\begin{aligned}
& \left(\frac{1}{r} \frac{\partial}{\partial r}\left(r \frac{\partial}{\partial r}\right)-\left(s^{2}+\omega^{2}\right)-\frac{1}{r^{2}}\left(n^{2}+1\right)\right) H_{n}=-\frac{\varepsilon_{n}}{\pi r} \delta\left(r-r^{\prime}\right), \\
& \varepsilon_{0}=\frac{1}{2}, \varepsilon_{n}=1, n \geq 1
\end{aligned}
$$

For $r \neq r^{\prime}$ it is obvious that the general solution to (25) will be a modified Bessel function of order $m= \pm \sqrt{n^{2}+1}$; thus $m$ is not an integer in general , and this suggests that one should perhaps begin with the $\theta$-independent solutions to (23) first. Easily, they would be the $n=0$ solutions of eq.(24) a general version of which is

$$
H_{0}\left(r, r^{\prime}\right)=c\left(r^{\prime}\right) I_{1}(k r)+d\left(r^{\prime}\right) K_{1}(k r), k^{2}=s^{2}+\omega^{2}
$$

with $I_{1}(k r), K_{1}(k r)$ being the modified Bessel functions of order 1 .

Moving on, Ref.6 helps to get a sharper version of (25) namely,

$$
H_{0}\left(r, r^{\prime}\right)=\theta\left(r^{\prime}-r\right) I_{1}(k r) K_{1}\left(k r^{\prime}\right)+\theta\left(r-r^{\prime}\right) K_{1}(k r) I_{1}\left(k r^{\prime}\right)
$$

with $\theta(x)$ the usual Heaviside function. Eq.(27) is the $n=0$ version of

$$
H_{n}\left(r, r^{\prime}\right)=\theta\left(r^{\prime}-r\right) I_{m}(k r) K_{m}\left(k r^{\prime}\right)+\theta\left(r-r^{\prime}\right) K_{m}(k r) I_{m}\left(k r^{\prime}\right), m=\sqrt{n^{2}+1}
$$

One can now fill in some of the steps now that have been passed over for eq.(24) et seq.; we have, following Ref.6 used the expansions

$$
\begin{gathered}
\delta\left(\theta-\theta^{\prime}\right)=\frac{1}{2 \pi}+\frac{1}{\pi} \sum_{1}^{\infty} \cos \left[n\left(\theta-\theta^{\prime}\right)\right] \\
H=e^{-i s z-i \omega x_{0}} \sum_{0}^{\infty} H_{n}\left(r, r^{\prime}\right) \varepsilon_{n} \cos \left[n\left(\theta-\theta^{\prime}\right)\right], \varepsilon_{0}=\frac{1}{2}, \varepsilon_{n}=1, n \geq 1
\end{gathered}
$$

to obtain the results given in eqs.(27) and (28); using (27) in (23) thus gets a $\theta-$ independent form of $\chi_{-1}$ which can now yield the $\tau_{k}, k \geq 1$ from the solution of the first order partial differential equations given in eqs.(6) and(7). This will be presented in detail elsewhere.

\section{References}

1. Gopinath Kamath, Cylindrical symmetry. An aid to calculating the zeta function in $3+1$ dimensional curved space, PoS ICHEP2016, 791(2016)

2. S. G. Kamath, A Derivation of the Scalar Propagator In A Planar Model in Curved Space, AIP Conf. Proc. 1246, 174 (2010) 
3. S. Deser, R. Jackiw and G.'tHooft, Three-dimensional Einstein gravity: Dynamics of Flat space, Ann.Phys.152, 220(1984)

4. G. Clement, Stationary solutions in three - dimensional general relativity, Int.J.Theor. Phys. 24, 267 (1985)

5. See eq.(20) in Clement,Ref.5

6. See for example, Dean G. Duffy, Green's Functions with Applications, Chapman and Hall/CRC(2001), Florida. 